\title{
Design of bistable gold@spin-crossover core-shell nanoparticles showing large electrical responses for the spin switching
}

Ramón Torres-Cavanillas ${ }^{\S}$, Roger Sanchis-Gual ${ }^{\S}$, Julien Dugay, Marc Coronado-Puchau*, Mónica Giménez-Marqués* and Eugenio Coronado*

R. Torres-Cavanillas, R. Sanchis-Gua, Dr. J. Dugay, Dr. M. Coronado-Puchau, Dr. M. Giménez-Marqués and Prof. E. Coronado

Instituto de Ciencia Molecular (ICMol), Universidad de Valencia, c/ Catedrático José Beltrán 2, Paterna, 46980, Spain.

E-mail: marc.coronado@uv.es, monica.gimenez-marques@uv.es, eugenio.coronado@uv.es

${ }^{\S} \mathrm{R}$. Torres-Cavanillas and R. Sanchis-Gual contributed equally to this work.

Keywords: core/shell nanoparticles, molecular electronics, molecular bistability, spincrossover, molecular switching devices

A simple protocol to prepare core-shell gold@spin-crossover (Au@SCO) nanoparticles (NPs) based on the $1 \mathrm{D}$ spin-crossover $\left[\mathrm{Fe}(\mathrm{Htrz})_{2}(\operatorname{trz})\right]\left(\mathrm{BF}_{4}\right)$ coordination polymer is reported. The synthesis relies on a two-step approach consisting on a partial surface ligand substitution of the citrate-stabilized Au NPs followed by the controlled growth of a very thin layer of the SCO polymer. As a result, colloidally stable core@shell spherical NPs of $19 \mathrm{~nm}$ in size exhibiting a narrow distribution in sizes have been obtained, revealing a switchable SCO shell of $c a .4 \mathrm{~nm}$. Temperature-dependent charge transport measurements of an electrical device based on assemblies of these $\mathrm{Au} @ \mathrm{SCO}$ NPs display well-defined, reproducible and sharp thermal hysteresis loops in the conductance near room temperature. This device is characterized both, by a large change in conductance upon spin state switching, and a remarkable transition abruptness, as compared with other memory devices based on the pristine SCO NPs. As a result, the sensitivity of the device to the spin transition is dramatically improved, with values for the ON/OFF ratio which are an order of magnitude better than the best ones obtained in previous SCO devices. 
An appealing example of molecular switching is provided by spin-crossover (SCO) compounds. These molecular-based materials present the ability to switch between two electronic states, namely the low spin (LS) and the high spin (HS) states under the influence of external stimuli, such as temperature, pressure, light, electric field or guest molecules. ${ }^{1}$ This spin transition leads to reversible changes in their optical, magnetic, mechanical and electrical properties, making them very promising as molecular switches, ${ }^{2}$ optoelectronic devices, ${ }^{3}$ actuators $^{4}$ and sensors. ${ }^{5}$

Of particular interest are those SCO materials displaying a hysteretic behaviour in their spin transition since they can be useful as components of non-volatile memory devices. ${ }^{6}$ In order to read out the spin state in these devices, materials with large electrical responses, preferably displaying wide hysteresis loops occurring near room temperature, are required. However, the SCO electronic devices reported to date have typically shown a gradual hysteresis in the conductance as well as very low electrical responses, owing to the insulating nature of the SCO material. ${ }^{7,8}$ This situation becomes even more complex when the SCO system is downscaled to the nanometer range since, under these circumstances, the hysteretic behaviour observed in bulk is generally lost upon reducing the size of the nanoparticles. ${ }^{9}$

Here the preparation of SCO-based devices presenting an abrupt transition in the conductance (occurring within a few Kelvin) and accompanied by a large electrical response in the spin switching is undertaken. Our strategy to reach this goal consists of designing core-shell nanoparticles (NPs) formed by a metallic Au core and a SCO shell. Despite the extensive works performed on core-shell NPs, ${ }^{10-12}$ nanostructures formed by a metallic core and a molecular SCO shell are unprecedented. In addition, as compared to previous examples that combine SCO entities and conducting metals, ${ }^{13-17}$ the core-shell configuration is expected to confront the insulating SCO behaviour. In these hybrid nanostructures, the appropriate selection of the SCO material should warrant the maintenance of an abrupt thermal hysteresis at the nanoscale, 
while the metallic core is expected to make these novel nanostructures more conductive. As SCO material the system of choice has been the well-known iron(II)-triazole coordination polymer of formula $\left[\mathrm{Fe}(\mathrm{Htrz})_{2}(\operatorname{trz})\right]\left(\mathrm{BF}_{4}\right)(\mathrm{Htrz}=1,2,4$-triazole $) .{ }^{18,19}$ This $\mathrm{SCO}$ material exhibits large and abrupt thermal hysteresis occurring near room temperature. Furthermore, its well-established miniaturization protocol, ${ }^{20-22}$ enables the maintenance of the cooperative spin transition features in NPs as small as $4 \mathrm{~nm} .{ }^{23}$ These NPs have already been integrated into electronic devices, showing a detectable conductivity change during the SCO transition. Thus, single NP devices (NP mean size of $10 \mathrm{~nm}$ ) showed an ON/OFF ratio in the conductivity of $c a$. 2. This performance is significantly improved in electronic devices based on 2D assemblies of SCO NPs for which the ON/OFF ratio increases up to a value of $c a .300 .^{24}$ In this context, the only attempt to combine metallic Au NPs with SCO molecules for the fabrication of molecular devices resulted in 2D arrays of SCO-functionalized $\mathrm{Au}$ NPs exhibiting however minor switching performances. $^{25}$

To synthesize the core-shell NPs a straightforward growth protocol that coats spherical Au NPs with a thin shell of Fe-triazole SCO material has been developed (Figure 1). First, citratestabilized gold NPs of $12.4 \pm 1.0 \mathrm{~nm}$ were synthesized following the Turkevich method. ${ }^{26}$ Then, the growth of the SCO complex around the Au NPs was performed following a two-step approach which consists on the partial ligand substitution of the gold surface agent, citrate, by the ligand triazole (Htrz) and the subsequent growth of the SCO polymer by controlled addition of the precursors (see Supporting Information, SI, for experimental details). 
(i) Partial ligand substitution

(ii) SCO overgrowth
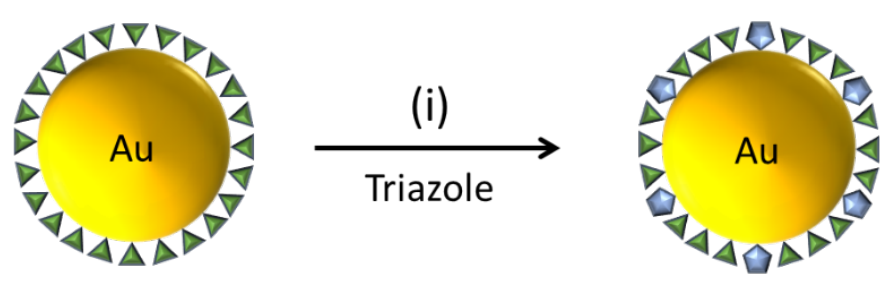

(ii)

$\mathrm{Fe}\left(\mathrm{BF}_{4}\right)_{2}$

Triazole
$\mathrm{Au}$

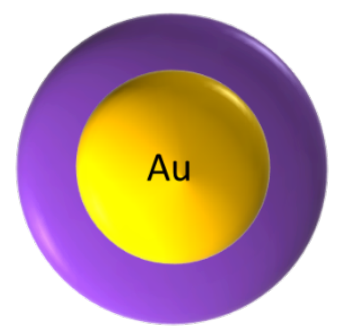

Figure 1. Schematic illustration of the steps involved in the preparation of Au@SCO NPs.

The first critical step occurs at the surface of stable Au NPs colloids, involving the partial ligand substitution of citrate capping agent by Htrz ligands. To do so, a diluted solution of Htrz ligand $(0.5 \mathrm{mM})$ was added into a stable suspension of citrate-stabilized Au NPs (0.16 mM). As a result, a partial ligand exchange occurred on the NP surface affording some nucleation sites that act as anchors for the subsequent growth of the SCO shell. Such a surface modification occurred thanks to the rather weak Au-citrate interaction ${ }^{27}$ and needed to be partial to maintain the colloidal stability of the NPs. In fact, a complete surface ligand substitution with Htrz can be achieved by using larger amounts of Htrz ligand, although in this case the Au NPs were not effectively stabilized and tended to aggregate Therefore, fine control of both the amount of Htrz ligands added as well as the reaction time were critical parameters to overgrow the shell while avoiding NP precipitation. This ligand exchange process was checked by X-Ray Photoemission spectroscopy (XPS), which showed the appearance of nitrogen atoms at the surface of the Au NPs after ligand exchange (Figure S1), in agreement with the presence of Htrz ligands.

The second key step of the protocol comprises the continuous growth of the SCO complex onto the pre-modified surface of the Au NPs (Figure 1) upon the controlled addition of the precursors. Thus, an aqueous solution of the $\mathrm{Fe}^{\mathrm{II}}$ precursor and the Htrz ligand were simultaneously added to the $\mathrm{Au}$ suspension at a constant rate $\left(4 \mathrm{~mL} \cdot \mathrm{h}^{-1}\right)$ in appropriate 
concentrations $(0.16 \mathrm{mM}$ and $0.5 \mathrm{mM}$, respectively), at room temperature and under stirring. It is worth mentioning that larger concentrations of either $\mathrm{Au} N \mathrm{NP}$ or $\mathrm{Fe}^{\mathrm{II}}$ precursors $(>0.3$ $\mathrm{mM}$ ) results in NP aggregation. After the complete addition of reagents, colloidally stable $\mathrm{Au} @ \mathrm{SCO}$ core-shell NPs were obtained. Interestingly and in contrast with the previously reported examples of SCO NPs, these Au@SCO NPs possess naked surfaces (i.e., free from any organic moiety). This feature may result particularly convenient to enhance the interactions between the SCO shells of neighboring NPs or to conduct post-synthetic modification on the surface of these NPs. Transmission Electronic Microscopy (TEM) images evidenced uniform $\mathrm{Au} @$ SCO NPs with an overall diameter of $19.4 \pm 1.9 \mathrm{~nm}$ and thin SCO shells of $3.6 \pm 1.0 \mathrm{~nm}$ (Figure 2.a and S5). A clear core@shell structure was distinguished in all the NPs, demonstrating the formation of an individual coating rather than a less controlled composite made of Au NPs embedded on SCO matrices. This narrow size distribution was also confirmed by Dynamic Light Scattering (DLS) measurements performed in water suspensions of $\mathrm{Au} @$ SCO NPs, which indicates a hydrodynamic diameter of $18 \pm 5 \mathrm{~nm}$ (Figure S2), dismissing the presence of aggregates. The SCO overgrowth was monitored by means of the particle size (Figure S3) showing a continuous growth upon addition of reagents. Z-potential analysis was used to assess the colloidal stability of the suspensions (Table S1), showing a strong modification from $-40 \pm 6 \mathrm{mV}$ to $-28 \pm 4 \mathrm{mV}$ for the $\mathrm{Au} @$ citrate and the $\mathrm{Au} @ \mathrm{SCO} \mathrm{NPs}$, respectively. Energy-dispersive X-ray spectroscopy (EDX) was used to estimate the metallic composition of the Au@SCO NPs (Figure S4) showing a clear core@shell distribution with an estimated ratio of $\mathrm{Au} / \mathrm{Fe}_{0.58}$. A more accurate analysis of the metal content was performed by Inductively Coupled Plasma Optical Emission Spectrometry (ICP-OES), where the relative (atomic) amount was found to be $\mathrm{Au} / \mathrm{Fe}_{0.62}$. X-ray diffraction analysis of the powdered materials (Figure S6) was performed in an attempt to characterize the chemical structure of the shell. The diffraction pattern of the Au@SCO NPs exhibited a unique peak at $38.2^{\circ}$ 
characteristic of metallic gold, whereas the expected peaks for the $\left[\mathrm{Fe}(\mathrm{Htrz})_{2}(\operatorname{trz})\right]\left(\mathrm{BF}_{4}\right)$ compound were not observed. ${ }^{28}$ This absence could be due to the small thickness of the SCO shell, which may be beyond the detection limit of the X-ray diffractometer, as well as to the large electron density of the crystalline Au cores, which can screen the signal coming from the SCO component. ${ }^{13}$

The optical properties of these hybrid NPs are reported in Figure $2 b$ and compared with the bare gold nanoparticles. It is observed that the localized surface plasmon resonance (LSPR) of Au NPs, which exhibits a maximum at $518 \mathrm{~nm}$, is gradually red-shifted during shell growth up to $530 \mathrm{~nm}$ in the Au@SCO NPs (see Figure S3). This plasmon shift was visually detected by a colour change in the suspension from red to pink, evidencing the high sensitivity of the LSPR to the presence of a dielectric SCO shell covering the Au NPs. In addition, a subsequent increase of the plasmon band intensity was also observed. Similar behaviour has been recently reported on a system based on Au NPs decorated with SCO molecules ([Fe(AcS$\left.\left.\mathrm{BPP})_{2}\right]\left(\mathrm{ClO}_{4}\right)_{2}\right) .{ }^{25}$ The mechanism at play may have its origin in the change of the dielectric properties at the Au NP surface upon the over-growth of the SCO shells around. ${ }^{29,30}$
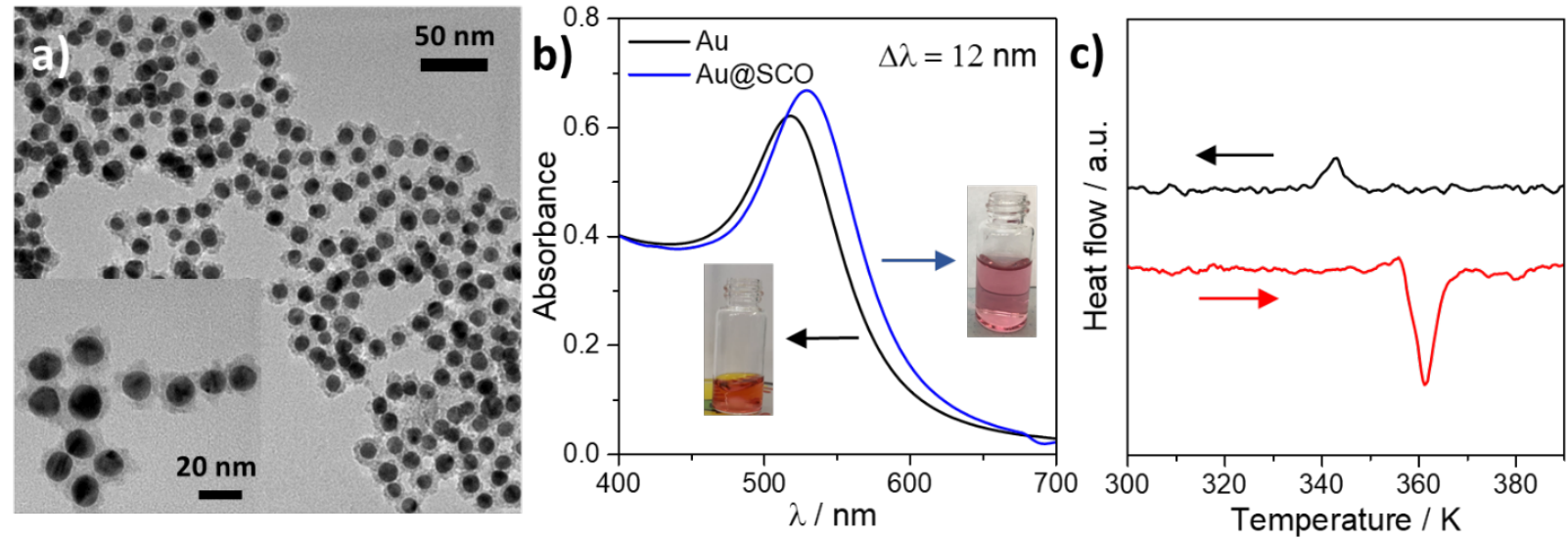

Figure 2.a) TEM image of Au@SCO NPs. Inset: HR-TEM image of Au@SCO NPs. b) Spectra of Au and Au@SCO NPs in the visible region. c) DSC curves of Au@SCO; the red line indicates the heating mode and the black line represents the cooling mode. 
The occurrence of a spin transition in the hybrid NPs was unambiguously confirmed by Differential Scanning Calorimetry (DSC) measurements that show two different peaks at 361 and $342 \mathrm{~K}$ in heating and cooling modes, respectively (Figure 2.c). This result is in good agreement with the already reported spin transition values for $4 \mathrm{~nm}$ NPs of the same SCO compound (367 and $343 \mathrm{~K}$ in heating and cooling mode, respectively). ${ }^{23}$ Three temperature cycles were carried out to probe the stability of the transition (Figure S7). Essentially, a remarkable hysteretic behaviour remains in the core@shell nanostructure despite the reduced thickness of the SCO shell, proving the strong cooperativity in the thermal spin switching.

We can take advantage of this cooperative spin transition to prepare memory devices in which the spin state is thermally addressed and electrically detected through temperature-dependent transport measurements. Thus, the hybridAu@SCO NPs were deposited into electrical devices consisting of interpenetrated "fingers" (10 $\mu \mathrm{m}$ gap) following a previously reported deposition process. $^{7}$ SEM images (Figure 3) and AFM characterization (Figure S8) performed after deposition and transport measurements revealed a dense and thin assembly of NPs between the electrodes, which remain stable without evidence of coalescence.
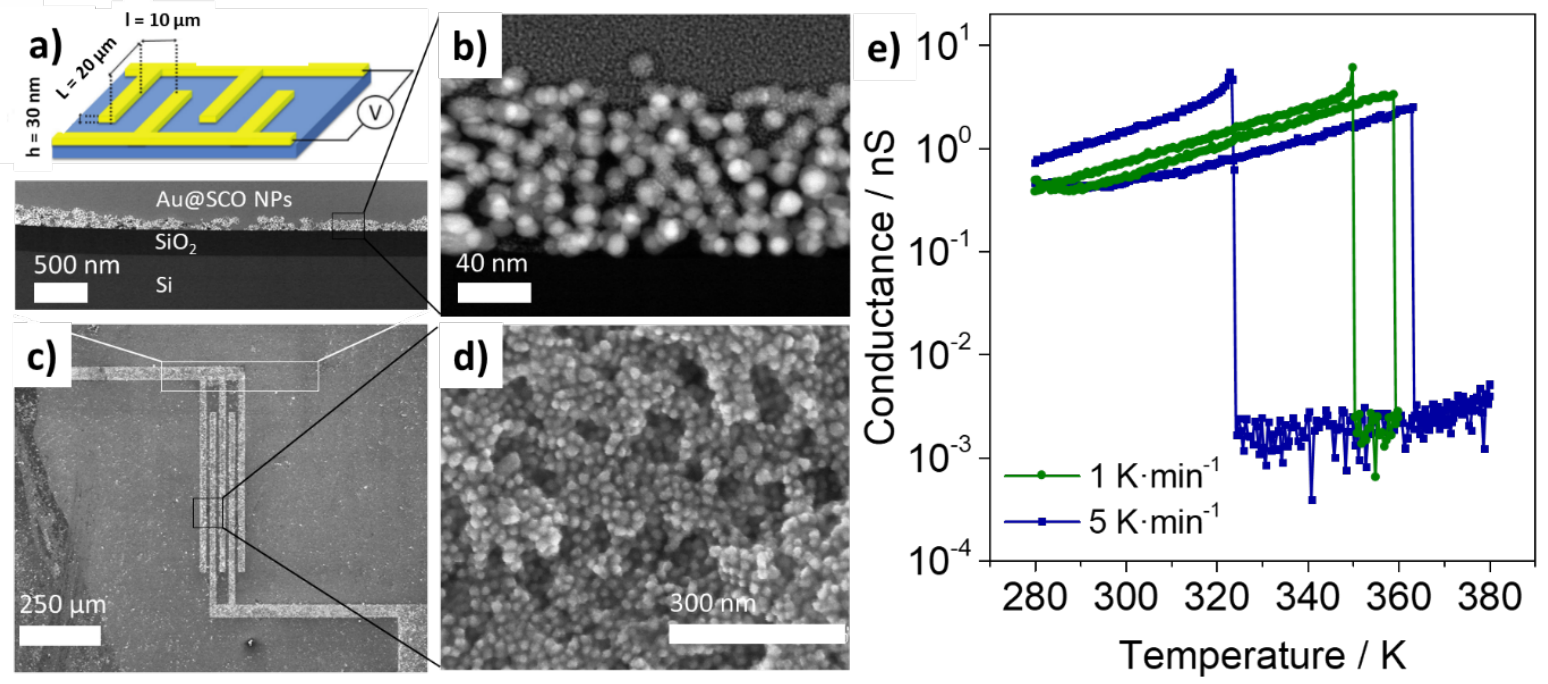
Figure 3. a) Scheme and dimensions of the electrodes and a, b) Scanning Transmission Electron Microscope cross-sectional micrographs of the devices. c, d) Scanning Electron Microscope (SEM) micrograph of the $\mathrm{Au} @ \mathrm{SCO}$ NPs trapped between Au finger-like electrodes. e) Thermal variation of the electrical current for the applied voltage $\mathrm{V}=2 \mathrm{~V}$ in the heating and cooling modes for two different scan rates ( 1 and $5 \mathrm{~K} \cdot \mathrm{min}^{-1}$ in green and blue, respectively).

Current-voltage (I-V) characteristics before and after deposition of Au@SCO NPs in the device were first compared, revealing a clear current increase that can be attributed to the presence of NPs in the gap (Figure S9). Then, temperature-dependent charge transport measurements were performed continuously recording the current at a fixed bias voltage of $\mathrm{V}=2 \mathrm{~V}$, while ramping the temperature at different speed rates (Figure S10). ${ }^{31}$ Figure 3.e depicts well-defined thermal hysteresis loops in the conductance for two temperature speed rates $\left(1\right.$ and $5 \mathrm{~K} \cdot \mathrm{min}^{-1}$ for green and blue curves, respectively). Essentially, the transition abruptness and the relative change in conductance are both maintained, evidencing reproducibility. I-V characteristics recorded at $350 \mathrm{~K}$ (i.e., within the thermal hysteresis region) in both the heating and cooling modes reveal high and low-conducting states, which can be attributed to the LS and HS states, respectively (Figure S11). Hence, a low conducting HS state is observed in $\mathrm{Au} @ \mathrm{SCO}$, in good agreement with previous studies performed in related SCO systems. ${ }^{7,24,25,32}$ In addition, a typical widening of the hysteresis loop is observed upon increasing the temperature speed rates, ${ }^{33}$ with a stronger dependence of $T^{\downarrow}{ }_{1 / 2}$ as compared with $T^{\uparrow}{ }_{1 / 2}$ values (Figure S10). Accordingly, such a dynamic behaviour affords a maximum value of $70 \mathrm{~K}$ for the hysteresis width at the fastest scan rate of $10 \mathrm{~K} \cdot \mathrm{min}^{-1}$. This value is much higher than those reported in similar SCO devices. ${ }^{34}$ Even more interesting is to observe that the transition efficiency in the electrical current occurs over ca. $1 \mathrm{~K}$ both in heating and cooling modes, and is also maintained upon applying different scan rates (i.e., $\left.1-10 \mathrm{~K} \cdot \mathrm{min}^{-1}\right)$. Such a remarkable abruptness results particularly significant considering the rather thin SCO shells $(c a .4 \mathrm{~nm})$ and the fact that miniaturization of SCO 
materials typically affords gradual and incomplete SCO transitions accompanied with a reduction of the hysteresis. The unprecedented sharp transition in the conductance can be explained by considering the coexistence of several factors, namely i) the preservation of thermal bistability in small SCO nanoparticles $(4 \mathrm{~nm})$ of the Fe-triazole series; ${ }^{23}$ ii) a reinforcement of the elastic interactions occurring at the core-shell interfaces because of the reduced sizes of the NPs ${ }^{35}$ and iii) the excellent thermal conductivity of the Au NP centres that are expected to provide a homogeneous and efficient heat transfer. All these aspects, operating either independently or synergistically, could render the observed fast and efficient spin transition.

Another remarkable feature is the high conductance values observed in this Au@SCO-based device, which are in the $\mathrm{nS}$ range despite the large electrode gap used (10 $\mu \mathrm{m}$, Figure 3.a). For comparison, an analog device based on SCO NPs of $16 \mathrm{~nm}$, stabilized by an organic surfactant and without the Au core was prepared. In this case, no electrical signal was recorded likely due to the insulating character of the sample. Such improvement in the conductance exhibited in the core-shell nanostructure with respect to that of the pure SCO NPs is attributed to the presence of the $\mathrm{Au}$ core, which largely enhances the conductance of these hybrid NPs, facilitating the transport through the NPs in the device. An important consequence of this feature, as far as the device performance is concerned, is that the conductivities associated with the two spin states exhibit ON/OFF ratios of about $5300 \pm 200$. These values are substantially larger than the previous record of $310 \pm 80$ reported by some of us in devices using 2D assemblies of $\left[\mathrm{Fe}(\mathrm{Htrz})_{2}(\operatorname{trz})\right]\left(\mathrm{BF}_{4}\right)$ NPs stabilized by an organic surfactant. ${ }^{24}$ This result implies a $1600 \%$ improvement in the electrical response of the spin switching.

To prove that electrical pathways are flowing through metallic dots separated by thin (insulating) shells rather than the hoping mechanism proposed in the pristine SCO NPs, we investigated the charge transport properties of another pair of combs in the Coulomb blockade 
regime (i.e., at much lower temperatures). Thus, the expected Coulomb blockade regime was evidenced by recording I-V characteristics from room temperature down to of about $10 \mathrm{~K}$. In such analysis, the resistance as a function of temperature underwent an increase of two to three orders of magnitude upon decreasing the temperature from room temperature down to $50 \mathrm{~K}$, which is typical of the Coulomb blockade in core-shell NP arrays (Figure S12). ${ }^{36}$ The overall observed temperature dependence of the conductance is non-Arrhenius-like but could be described regarding a percolation theory taking the disorder of the system into account. Indeed, a rather disordered NP film is observed (Figure 3) likely due to the actual distribution of sizes both, of the NP cores and shells as well as by off-set charges. More information about the theoretical model can be found in the supporting information. A comparison between model calculations and experimental results of the memory effect in the conductance versus temperature is available in the Figure S13 and S14. Importantly, this percolation model combined with a memory effect in the resistance versus temperature grasps the main features of the data, including the presence and the clockwise direction of the hysteresis loops from above the spin-transition down to the Coulomb blockade regime.

In summary, we have reported a simple protocol to prepare stable colloidal suspensions of coreshell NPs formed by a spherical metallic Au core of $c a .12 \mathrm{~nm}$ surrounded by a thin shell of an insulating SCO coordination polymer, $4 \mathrm{~nm}$ thick. Interestingly, these hybrid nanostructures have displayed an abrupt spin transition with a pronounced memory effect in the conductance close to room temperature. This protocol is now being extended to other $\mathrm{Au} @ \mathrm{SCO}$ nanostructures presenting different core sizes and shapes, as well as different thicknesses for the SCO shell. Thanks to the metallic core, these core-shell NPs are much more conductive than the pristine SCO NPs. This feature has enabled a sharp improvement in the electrical detection of the spin state, with unprecedented ON/OFF ratios in the conductance of the two spin states (of the order of 5300). Because of all these properties, the Au@SCO nanostructures 
reported here are excellent platforms to develop highly sensitive switching devices based on the SCO phenomenon. A further development we can imagine is that of taking advantage of the plasmon properties of the Au core to get a light-induced spin transition at room temperature. In this case, the efficient absorption of light provided by the plasmonic NP should be sufficient to heat the SCO shell and to provoke the spin transition.

\section{Acknowledgments}

We acknowledge funding from the EU (COST Action MOLSPIN CA15128, ERC Advanced Grant Mol-2D 788222, and FET-OPEN COSMICS 766726), Spanish MINECO (Unit of Excellence "Maria de Maeztu" MDM-2015-0538 and Project MAT2017-89993-R co-financed by FEDER), and Generalitat Valenciana (Prometeo/2017/066). R.T.-C., R.S.-G. and M.G.-M. thank the Ministerio de Ciencia, Innovación y Universidades for the F.P.I., F.P.U. and Juan de la Cierva Incorporation fellowships, respectively. Authors thank Marta Galbiati, from ICMol, for experimental help, and Rodrigo Fernandez-Pacheco and Laura Casado, from LMA-INA

\section{References}

[1] (a) L. Cambi, A. Gagnasso, Atti Accad. Naz. Lincei 1931, 13, 809. (b) W. A. Baker, H. M.

Bobonich, Inorg. Chem. 1964, 3, 1184. (c) P. Gütlich, in Structure and Bonding, (Eds.: M. J. Clarke, J. B. Goodenough, P. Hemmerich, J. A. Ibers, C. K. Jörgensen, J. B. Neilands, D. Reinen, R. Weiss, R. J. P. Williams), Springer, Berlin, Heidelberg 1981, 83. (d) Spin Crossover in Transition Metal Compounds I-III (Eds: P. Gütlich, H. A. Goodwin), Springer, Berlin, Heidelberg 2004.

[2] F. Prins, M. Monrabal-Capilla, E. A. Osorio, E. Coronado, H.S.J. Van Der Zant, Adv. Mater. 2011, 23 (13), 1545.

[3] F. Guillaume, Y.A. Tobon, S. Bonhommeau, J.F. Létard, L. Moulet, E. Freysz, Chem. Phys. Lett. 2014, 604, 105.

[4] G. Molnár, L. Salmon, W. Nicolazzi, F. Terki, A. Bousseksou, J. Mater. Chem. C 2014, 2 (8), 1360 . 
[5] E. Coronado, M. Giménez-Marqués, G. Mínguez Espallargas, F. Rey, I.J. VitóricaYrezábal, J. Am. Chem. Soc. 2013, 135 (43), 15986.

[6] O. Kahn, C. Jay Martinez, Science 1998, 279 (5347), 44.

[7] A. Rotaru, J. Dugay, R.P. Tan, I.A. Guralskiy, L. Salmon, P. Demont, J. Carrey, G. Molnár, M. Respaud, A. Bousseksou, Adv. Mater. 2013, 25 (12), 1745.

[8] K.S. Kumar, M. Ruben, Coordination Chemistry Reviews 2017, 346, 176.

[9] (a) G. Molnár, S. Rat, L. Salmon, W. Nicolazzi, A. Bousseksou, Adv. Mater. 2018, 30 (5), 1703862. (b) F. Volatron, L. Catala, E. Rivière, A. Gloter, O. Stéphan, T. Mallah, Inorg. Chem. 2008, 47(15), 6584. (c) V. Martínez, L. Boldog, A. B. Gaspar, V. Ksenofontov, A. Bhattacharjee, P. Gütlich, J. A. Real, Chem. Mater. 2010, 22 (14), 4271.

[10] L. Catala, D. Brinzei, Y. Prado, A. Gloter, O. Stéphan, G. Rogez, T. Mallah, Angew. Chem. Int. Ed. 2009, 48 (1), 183.

[11] A.C. Felts, A. Slimani, J. M. Cain, M.J. Andrus, A.R. Ahir, K.A. Abboud, W. Meisel, K. Boukheddaden, D.R. Talham, J. Am. Chem. Soc. 2018, 140 (17), 5814.

[12] G. Maurin-Pasturel, J. Long, Y. Guari, Y., F. Godiard, M.G. Willinger, C. Guerin, J. Larionova, Angew. Chem. Int. Ed. 2014, 126 (15), 3953.

[13] D. Qiu, L, Gu, X. L. Sun, D. H. Ren, Z. G. Gu, Z. Li, RSC Adv. 2014, 4, 61313.

[14] L. Moulet, N. Daro, S. Mornet, N. Vilar-Vidal, G. Chastanet, P. Guionneau, Chem Commun 2016, 52 (90), 13213.

[15] I. Suleimanov, J. Sánchez Costa, G. Molnár, L. Salmon, A. Bousseksou, Chem. Commun. 2014, $50(86), 13015$.

[16] Z. H. Li, Y. X. Wang, W. K. Han, W. Zhu, T. Li, Z. Li, X. Ren, Z. G. Gu, New J. Chem. 2017, $41(18), 10062$.

[17] Y.A. Tobon, C. Etrillard, O. Nguyen, J.F. Létard, V. Faramarzi, J.F. Dayen, B. Doudin, D. M. Bassani, F. Guillaume, Eur. J. Inorg. Chem. 2012, 2 (35), 5837. 
[18] J.G. Haasnoot, V. Ger, L. G. Willem, Zeitschrift für Naturforschung B 1977, 32 (12) 1421.

[19] J. Krober, J. Audière, R. Claude, E. Codjovi, O. Kahn, J. G. Haasnoot, F. Grolière, C. Jay, A. Bousseksou, J. Linarès, F. Varret, A. Gonthier-Vassal, Chem. Mater., 1994, 6 (8), 1404. [20] E. Coronado, J. R, Galán-Mascarós, M. Monrabal-Capilla, J. García-Martínez, P. PardoIbañez, Adv. Mat. 2007, 19 (10), 1359.

[21] L. Moulet, N. Daro, C. Etrillard, J.F. Létard, A. Grosjean, P. Guionneau, Magnetochemistry 2016, 2 (1), 10.

[22] J. R. Galán-Mascarós, E. Coronado, A. Forment-Aliaga, M. Monrabal-Capilla, E. PinillaCienfuegos, M. Ceolin, Inorg. Chem. 2010, 49 (12), 5706.

[23] M. Giménez-Marqués, M. L. García-Sanz de Larrea, E. Coronado, J. Mater. Chem. C 2015, 3 (30), 7946.

[24] J. Dugay, M. Giménez-Marqués, T. Kozlova, H. W. Zandbergen, E. Coronado, H. S. J. Van der Zant, Adv. Mater. 2015, 27 (7), 1288.

[25] E. J. Devid, P. N. Martinho, M. V. Kamalakar, I. Šalitroš, Ú. Prendergast, J. F. Dayen, V. Meded, T. Lemma, R. González-Prieto, F. Evers, T.E. Keyes, M. Ruben, B. Doudin, S. J. Van Der Molen, ACS Nano 2015, 9 (4), 4496.

[26] J. Turkevich, Gold Bull. 1985, 18 (4), 125.

[27] J. W. Park, J. S. Shumaker-Parry, J. Am. Chem. Soc. 2014, 136 (5), 1907

[28] A. Urakawa, W. Van Beek, M. Monrabal-Capilla, J. R. Galán-Mascarós, L. Palin, M. Milanesio, J. Phys. Chem. C 2011, 115 (4), 1323.

[29] M. M. Miller, A. A. Lazarides, J. Phys. Chem. B 2005, 109 (46), 21556.

[30] K. M. Mayer, J. H. Hafner, Chem. Rev. 2011, 111 (6), 3828.

[31] C. Lefter, V. Davesne, L. Salmon, G. Molnár, P. Demont, A. Rotaru, A. Bousseksou, Magnetochemistry 2016, 2 (1), 18. 
[32] A. Holovchenko, J. Dugay, M. Giménez-Marqués, R. Torres-Cavanillas, E. Coronado, H. S. J. van der Zant, Adv. Mater. 2016, 28, 7228.

[33] (a) R. Kulmaczewski, J. Olguín, J.A. Kitchen, H. L. Feltham, G. N. Jameson, J. L. Tallon, S. Brooker, J. Am. Chem. Soc. 2014, 136 (3), 878. (b) R. G. Miller, S. Narayanaswamy, J. L. Tallon, S. Brooker, New J. Chem. 2014, 38 (5), 1932.

[34] Y. S. Koo, J. R. Galán-Mascarós, Adv. Mater. 2014, 26 (39), 6785.

[35] M. Mikolasek, G. Félix, W. Nicolazzi, G. Molnar, L. Salmon, A. Bousseksou, New. J. Chem. 2014, 38 (5), 1834.

[36] J. Dugay, R. P. Tan, M. Ibrahim, C. Garcia, J. Carrey, L. M. Lacroix, P. F. Fazzini, G. Viau, M. Respaud, Phys. Rev. B 2014, 89 (4), 041406. 


\section{Supporting Information}

Design of bistable gold@spin-crossover core-shell nanoparticles showing large electrical responses for the spin switching

Ramón Torres-Cavanillas ${ }^{\S}$, Roger Sanchis-Gual ${ }^{\S}$,Julien Dugay, Marc Coronado-Puchau*, Mónica Giménez-Marqués* and Eugenio Coronado*

\section{Table of Contents:}

Experimental section:

X-ray Photoemission Spectroscopy

Figure S1

Dynamic Light Scattering

Table S1

Figure S2-3

Transmission Electron Microscopy

Figure S4-5

X-Ray Powder Diffraction

Figure S6

Differential scanning calorimetry

Figure S7

Atomic Force Microscopy characterization

Figure S8

Transport measurement

Figure S9-12

Numerical calculation details

Figure S13-14

References 


\section{Experimental section}

Materials:

All chemical reagents were purchased and used without further purification: chloroauric acid (Sigma-Aldrich), sodium citrate tribasic dihydrate (Sigma-Aldrich), 1,2,4-triazole (SigmaAldrich), iron tetrafluoroborate hexahydrate (Sigma-Aldrich) and ultra pure water (18.2 M $\Omega$ ).

Au@SCO synthesis. Iron-triazole polymeric system was grown onto citrate-stabilized gold nanoparticles following a two-step approach. Gold nanoparticles of $12 \mathrm{~nm}$ were freshly prepared using the well-known Turkevich method ${ }^{1}$ and then washed with water to remove the excess of citrate in the solution. In a first step, a triazole aqueous solution ( $200 \mu \mathrm{L}, 0.5 \mathrm{mM})$ was added into the previously prepared Au NPs aqueous solution $(3 \mathrm{~mL}, 0.16 \mathrm{mM})$ under continuous stirring in air for 30 minutes. In a second step, iron tetrafluoroborate hexahydrate $(3 \mathrm{~mL}, 0.16 \mathrm{mM})$ and triazole $(3 \mathrm{~mL}, 0.5 \mathrm{mM})$ aqueous solutions were simultaneously added $\left(4 \mathrm{~mL} \cdot \mathrm{h}^{-1}\right)$ under stirring in air at room temperature. The obtained core-shell Au@SCO nanoparticles were then collected by centrifugation ( $8000 \mathrm{rpm}, 30 \mathrm{~min}$.) and easily re-dispersed in different solvents (water, ethanol, acetone, and chloroform).

UV/Vis Spectroscopy: UV-vis absorption spectra were recorded on a Jasco V-670 spectrophotometer in baseline mode from 400 to $800 \mathrm{~nm}$ range, using 1.000-cm-optical-path plastic cuvettes.

High-resolution transmission electron microscopy (HRTEM) and transmission electron microscopy (TEM): HRTEM studies were carried out on a Technai G2 F20 microscope operating at $200 \mathrm{kV}$ and TEM studies on a JEM-1010 operating at $100 \mathrm{kV}$. Samples were prepared by dropping suspensions on lacey formvar/carbon copper grids (300 mesh).

Zeta Potential (ZP) and Dynamic Light Scattering (DLS) measurements: ZP and DLS measurements were performed at room temperature with a Zetasizer Nano ZS instrument (Malvern Instruments Ltd.) of the as-synthesized suspension.

X-ray Photoelectron Spectroscopy (XPS): Samples were analyzed ex-situ at the X-ray Spectroscopy Service at the Universidad de Alicante using a K-ALPHA Thermo Scientific spectrometer. All spectra were collected using Al Ka radiation $(1486.6 \mathrm{eV})$, monochromatized by a twin crystal monochromator, yielding a focused X-ray spot (elliptical in shape with a major axis length of $400 \mu \mathrm{m}$ ) at $30 \mathrm{~mA}$ and $2 \mathrm{kV}$. The alpha hemispherical analyzer was operated in the constant energy mode with survey scan pass energies of $200 \mathrm{eV}$ to measure the whole energy band and $50 \mathrm{eV}$ in a narrow scan to selectively measure the particular elements. 
XPS data were analyzed with Avantage software. A smart background function was used to approximate the experimental backgrounds. Charge compensation was achieved with the system flood gun that provides low energy electrons and low energy argon ions from a single source.

Transport measurements: All transport measurements have been performed using a twoterminal device configuration in a temperature range of 10 to $380 \mathrm{~K}$ under a high vacuum. A Physical Properties Measurement System (Quantum Design PPMS-9). Electrical measurements have been performed in the PPMS chamber using a Keithley 2450 voltage source and a Keithley 6517b electrometer. Dielectrophoresis was used to trap Au@SCO NPs between the electrodes by powering a sinusoidal signal of $10 \mathrm{kHz}$ frequency and $10 \mathrm{~V}$ peakto-peak potential to the electrodes.

Inductively Coupled-Plasma Optical Emission Spectrometry (ICP-OES): ICP-OES analysis was conducted at the Universidad Complutense de Madrid (CAI de Técnicas Geológicas). Samples were digested in a mixture of hydrochloric and nitric acids using a high-pressure microwave oven.

$X$-Ray Powder Diffraction (XRPD): X-ray powder diffraction (XRPD) patterns were obtained with a PANalytical Empyrean X-ray diffractometer using copper radiation source $(\mathrm{Cu}-\mathrm{Ka}=$ $1.54178 \AA$ ) in the 5-40 region.

Differential Scanning Calorimetry (DSC): A Mettler Toledo DSC 821e equipped with a liquid nitrogen cryostat and a $200 \mathrm{~W}$ furnace working at $10^{\circ} \mathrm{C} / \mathrm{min}$ heating rate was used.

Measurements were collected in the $25-130^{\circ} \mathrm{C}$ temperature range in three different cycles. 

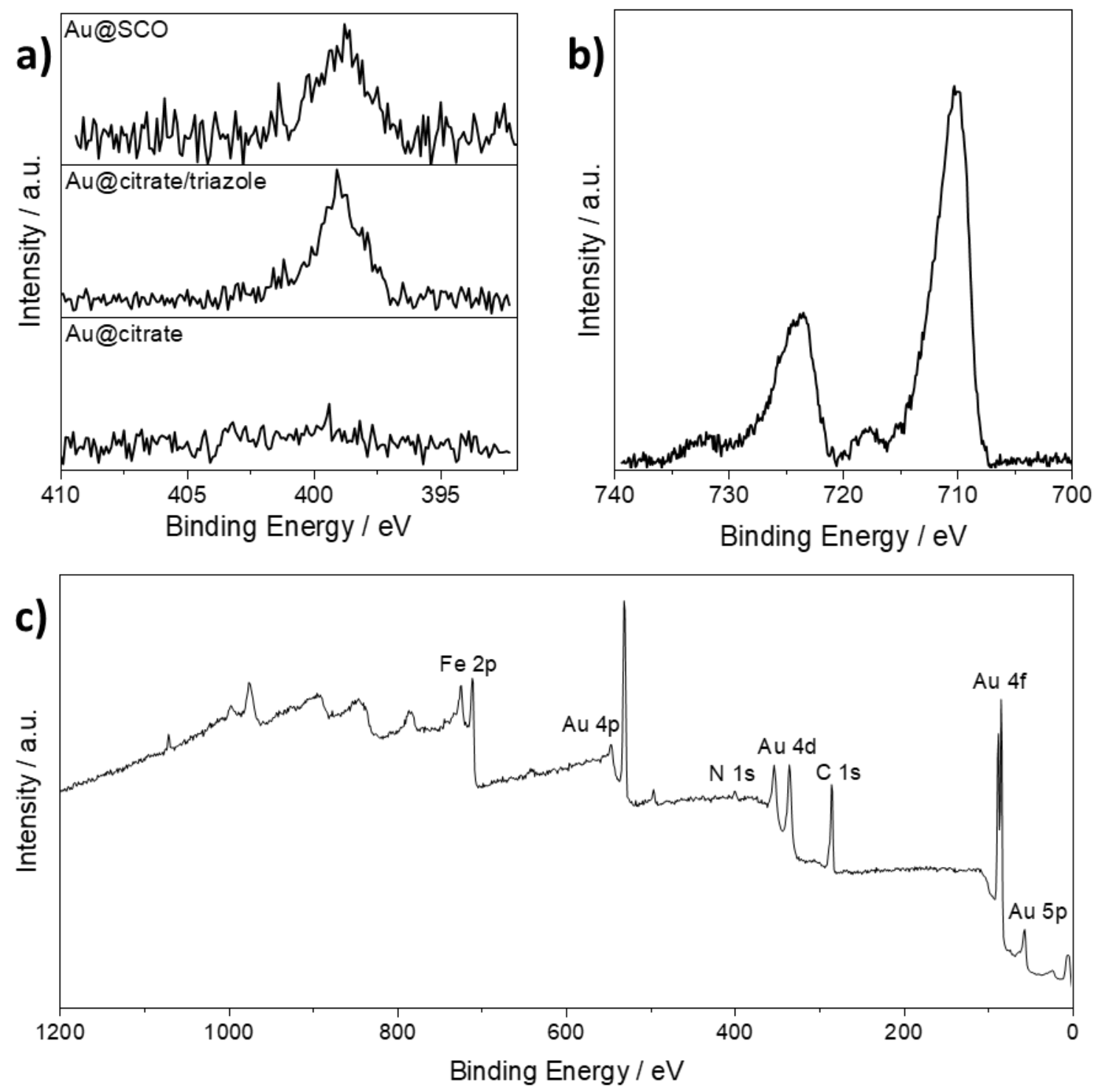

Figure S1. XPS spectra of a) N b) Fe and c) Survey for Au@SCO NPs.

After triazole exchange new peaks appear around $400 \mathrm{eV}$, associated with triazole N1S electrons, indicating the presence of the ligand at the gold surface. 


\section{Dynamic Light Scattering measurements}

Table S1: Zeta potentials of Au@citrate nanoparticles (NPs), Au@citrate/triazole NPs and Au@SCO NPs.

\begin{tabular}{lcc} 
Sample & Z-potential / mV & pH \\
\hline Au@citrate & $-40.0 \pm 0.6$ & 6.61 \\
Au@citrate/Trz & $-40.1 \pm 0.5$ & 6.77 \\
Au@SCO & $-28.1 \pm 1.4$ & 6.52
\end{tabular}

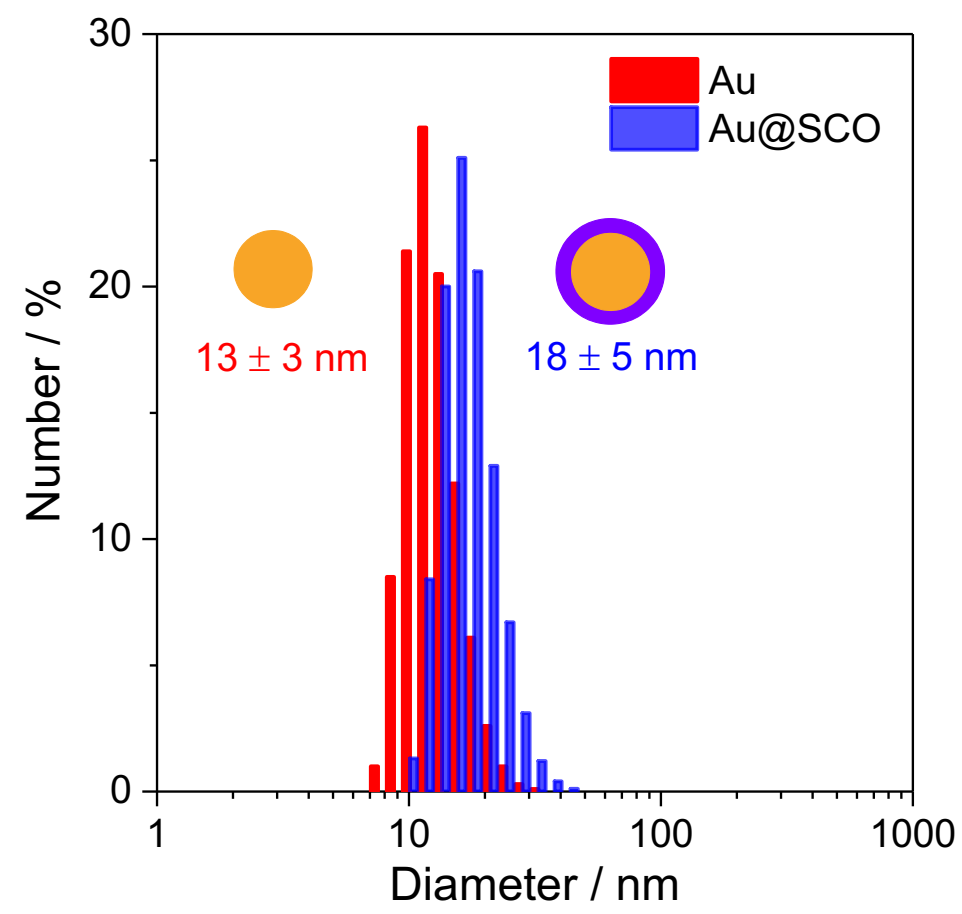

Figure S2. Dynamic Light Scattering plot for citrate-stabilized Au NPs and Au@SCO NPs. 


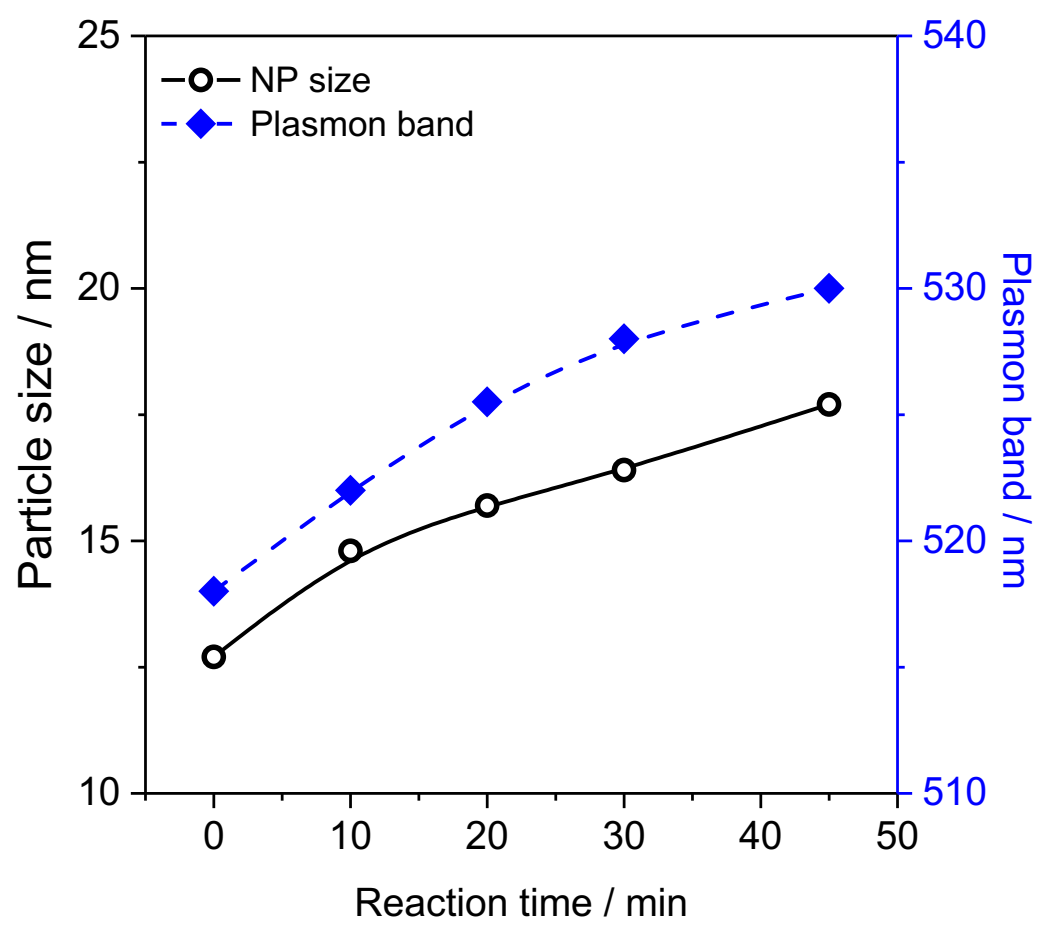

Figure S3. Evolution of the particle size and plasmon band position upon the addition of the precursors during the synthesis. 


\section{Transmission Electron Microscopy}
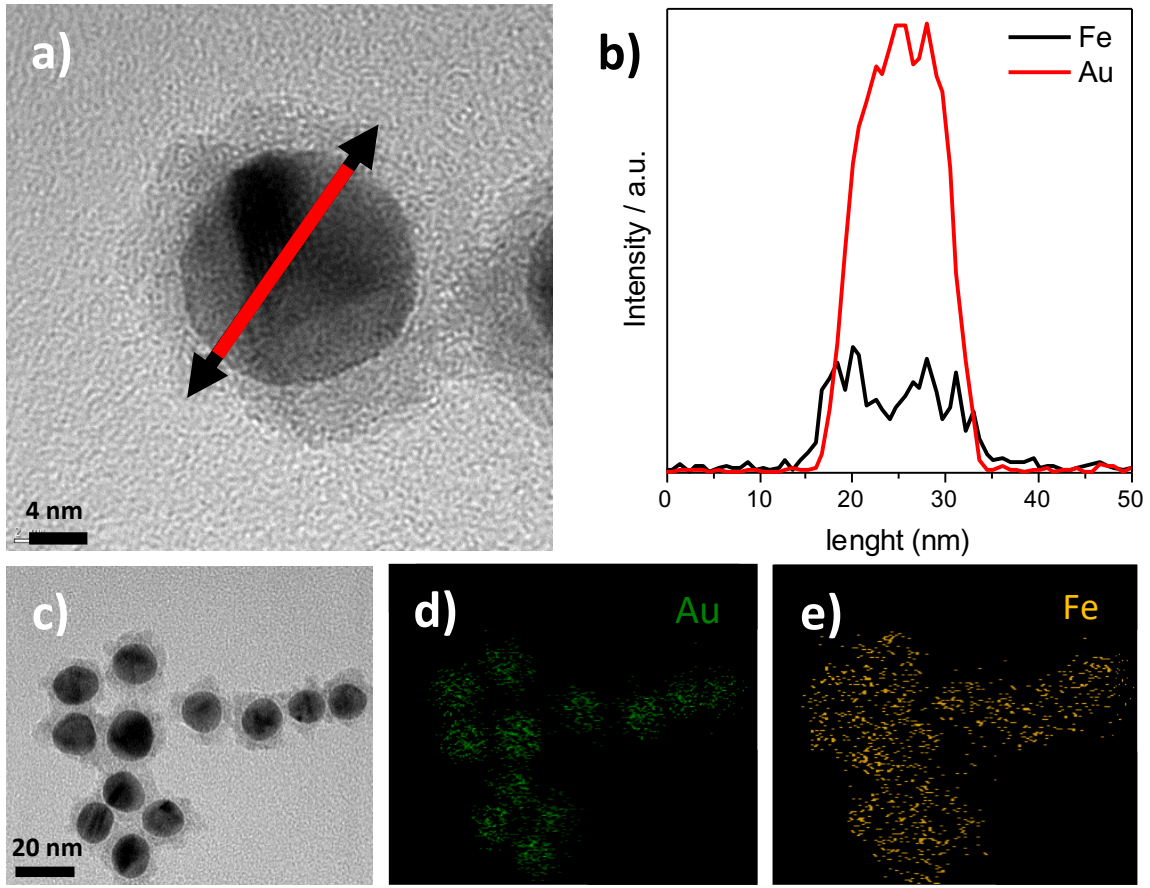

Figure S4. a) High-Resolution TEM image and b) EDX profile showing the presence of $\mathrm{Au}$ and $\mathrm{Fe}$ on a single NP; $\mathrm{c}-\mathrm{e}$ ) EDX mapping of the metals present in the heterostructure.

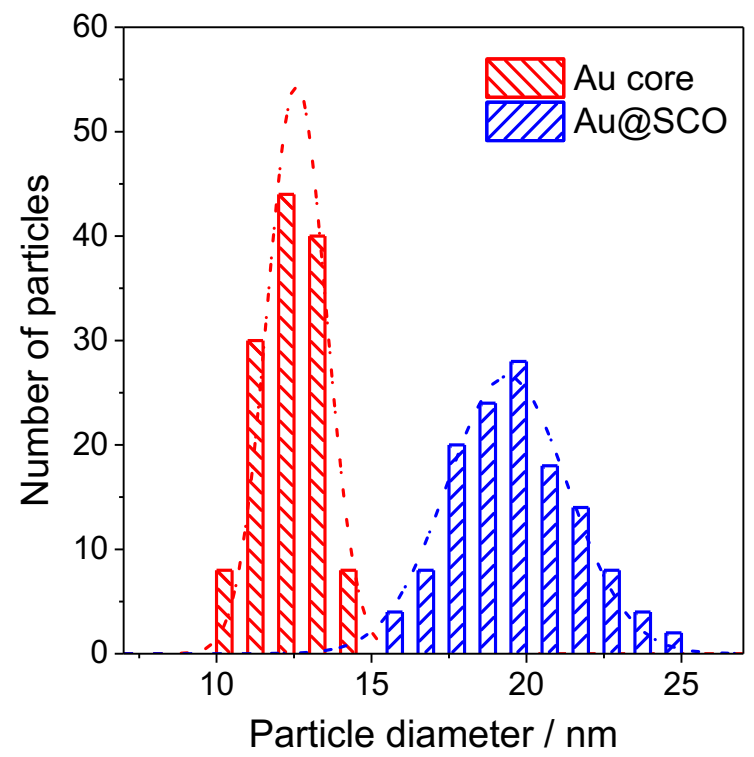

Figure S5. Histograms of the size distributions for the gold core and the overall nanoparticle diameter. 
X-Ray Powder Diffraction

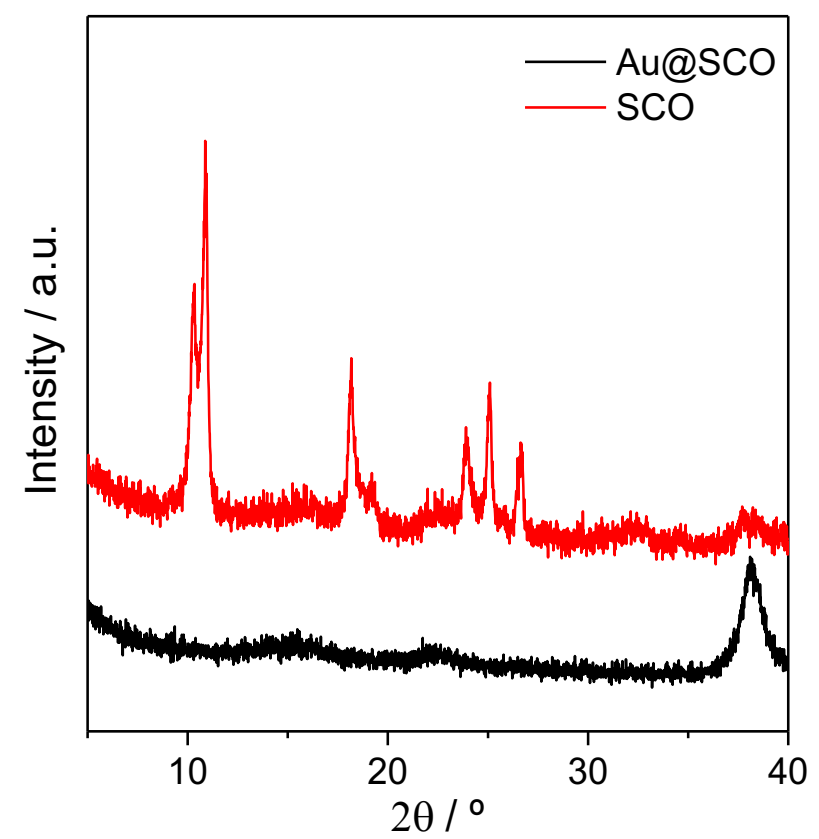

Figure S6. Room temperature XRPD for Au@SCO NPs (black) and pristine SCO (red) compound. 


\section{Differential Scanning Calorimetry}

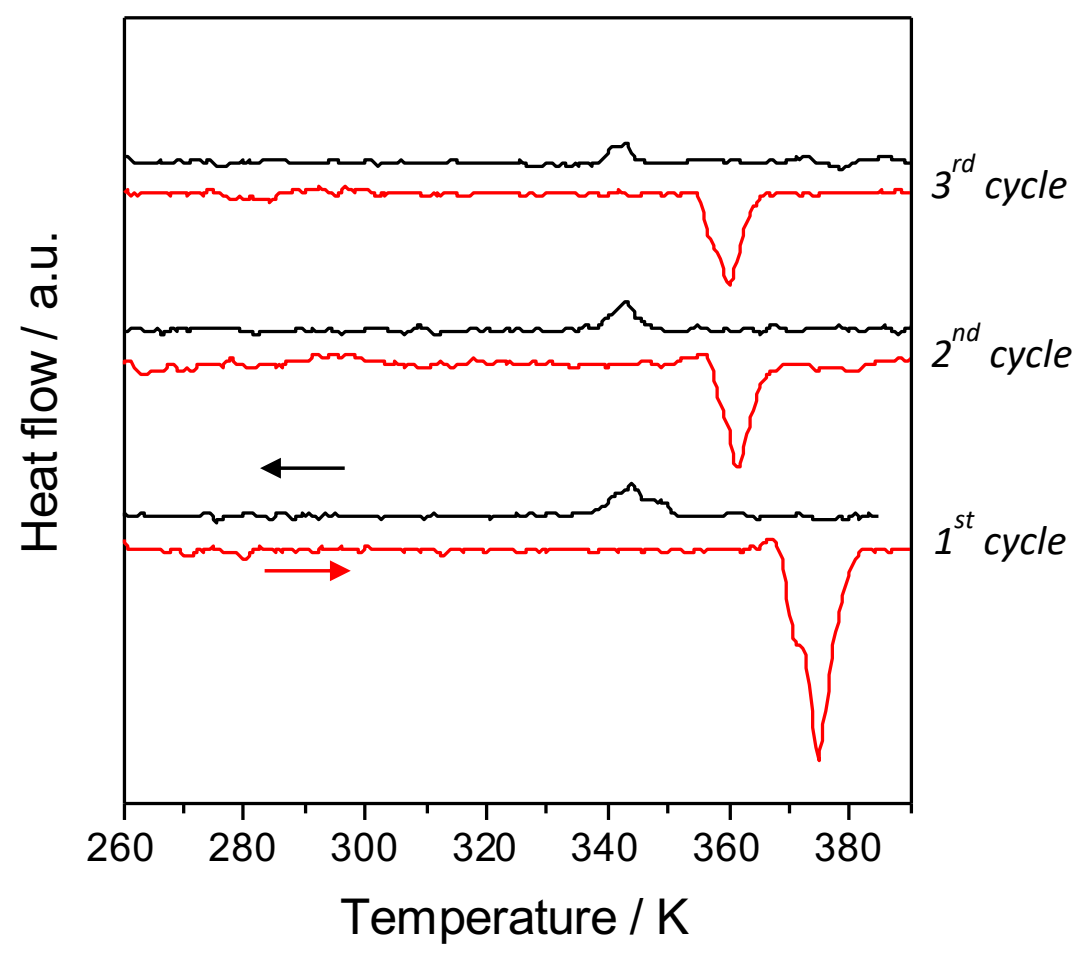

Figure S7. DSC curves of Au@SCO; the red line indicates the heating mode and the black line represents the cooling mode. 
Atomic Force Microscopy (AFM) characterization
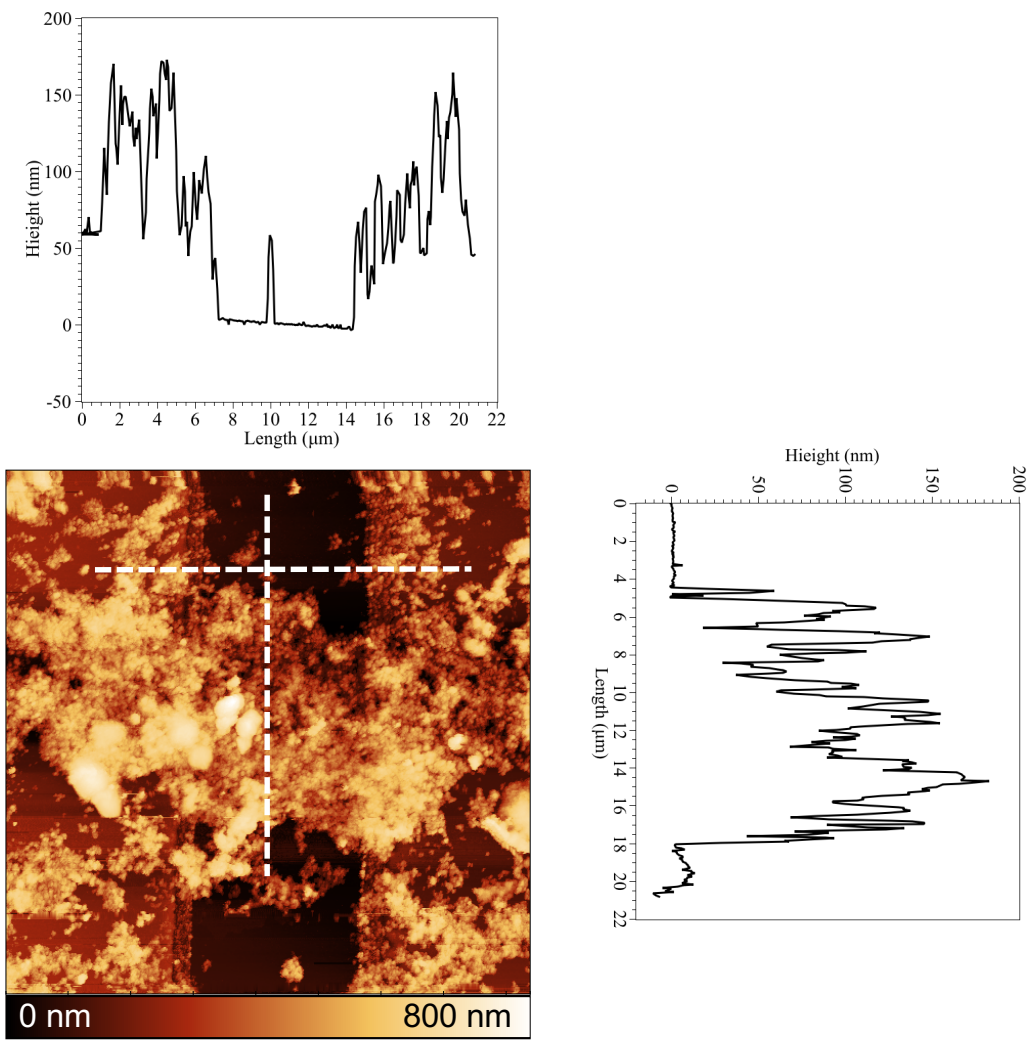

Figure S8 AFM image of a representative area of an assembly of Au@SCO NPs of a few layer thickness bridging source and drain electrodes. 


\section{Transport measurements}

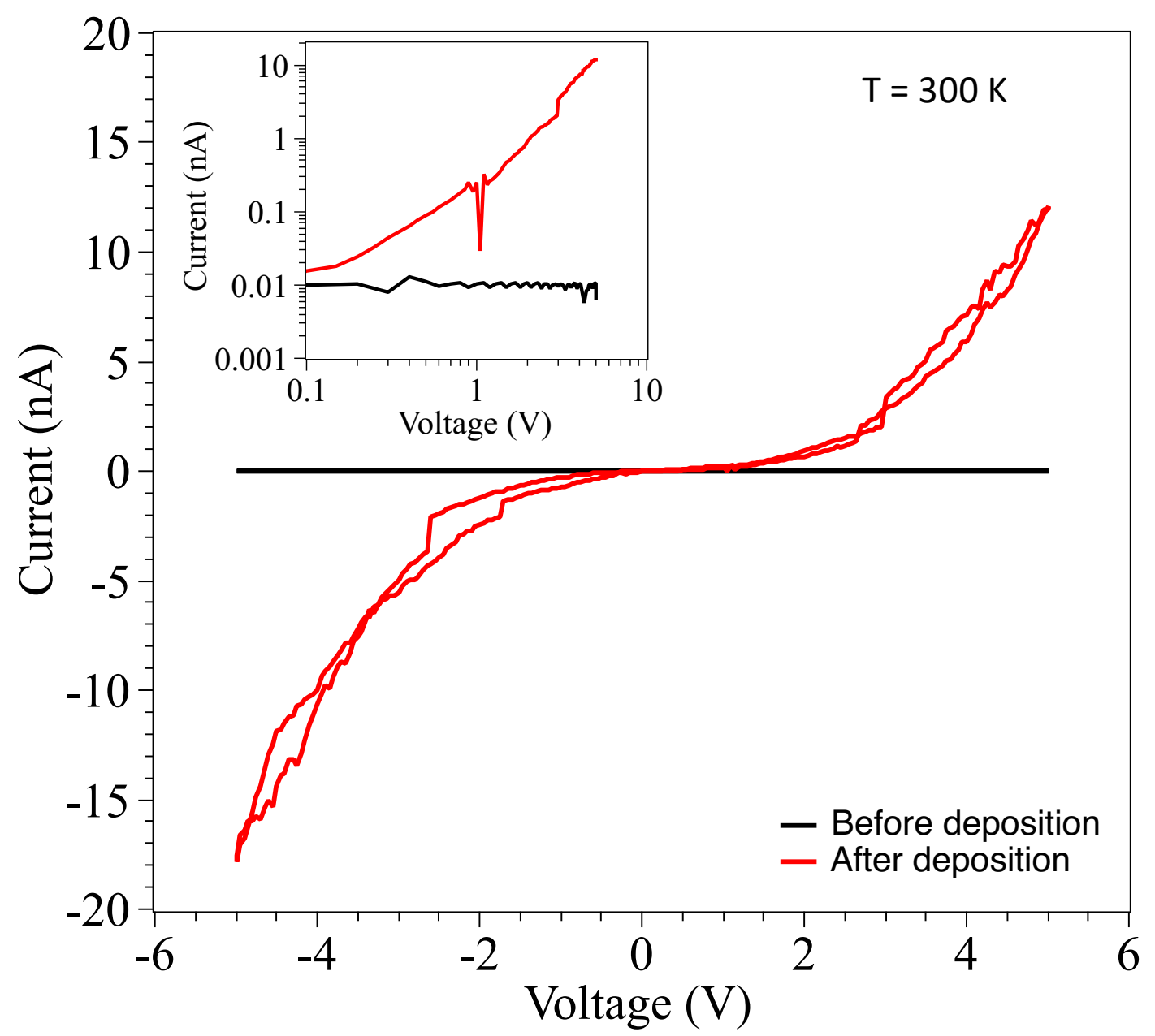

Figure S9. Room temperature current-voltage characteristics after deposition are compared with the characteristics before deposition. Changes in the transport-characteristics can then be attributed to the presence of hybrid Au@SCO NPs in the gap. 


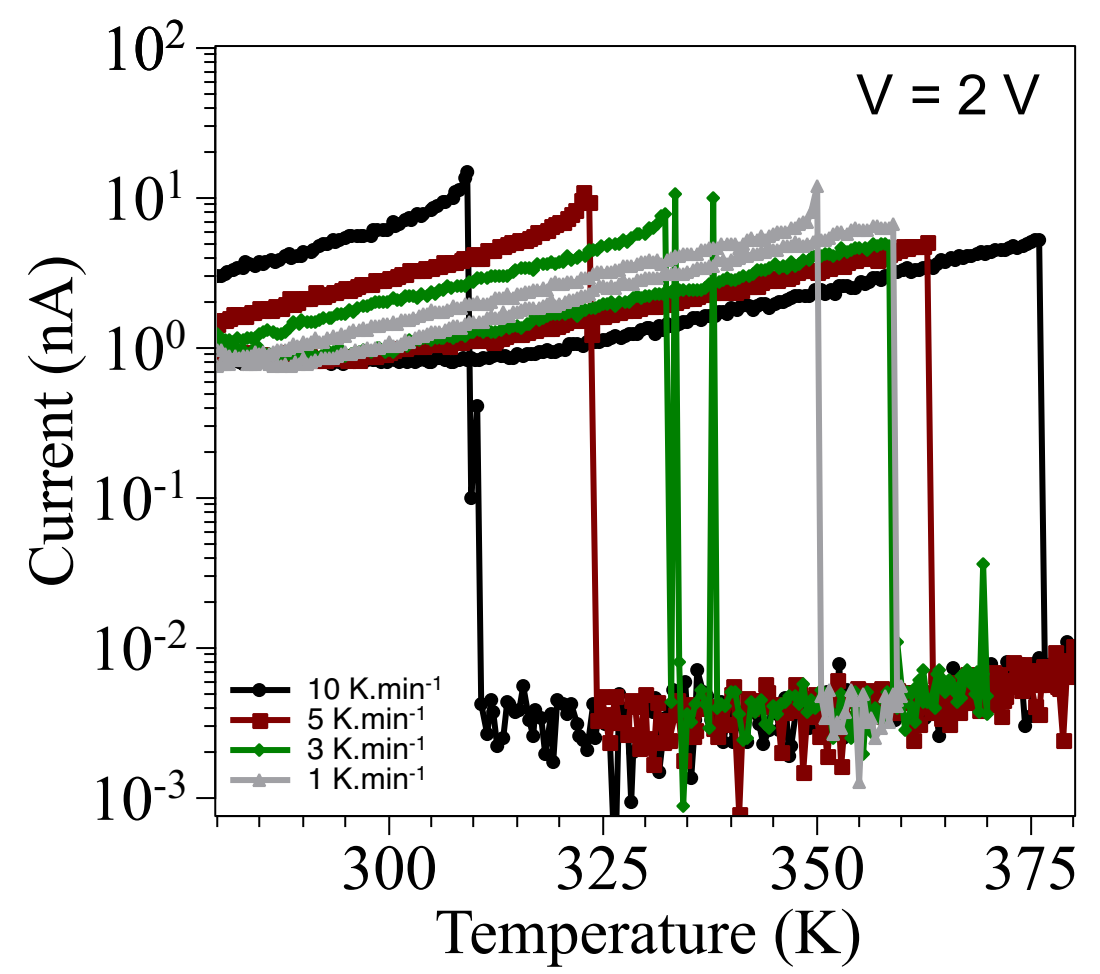

Figure S10. Thermal variation of the electrical current for the applied voltages $\mathrm{V}=2 \mathrm{~V}$ in the heating and cooling modes for various scan rates $\left(1,3,5\right.$ and $\left.10 \mathrm{~K} \cdot \mathrm{min}^{-1}\right)$.

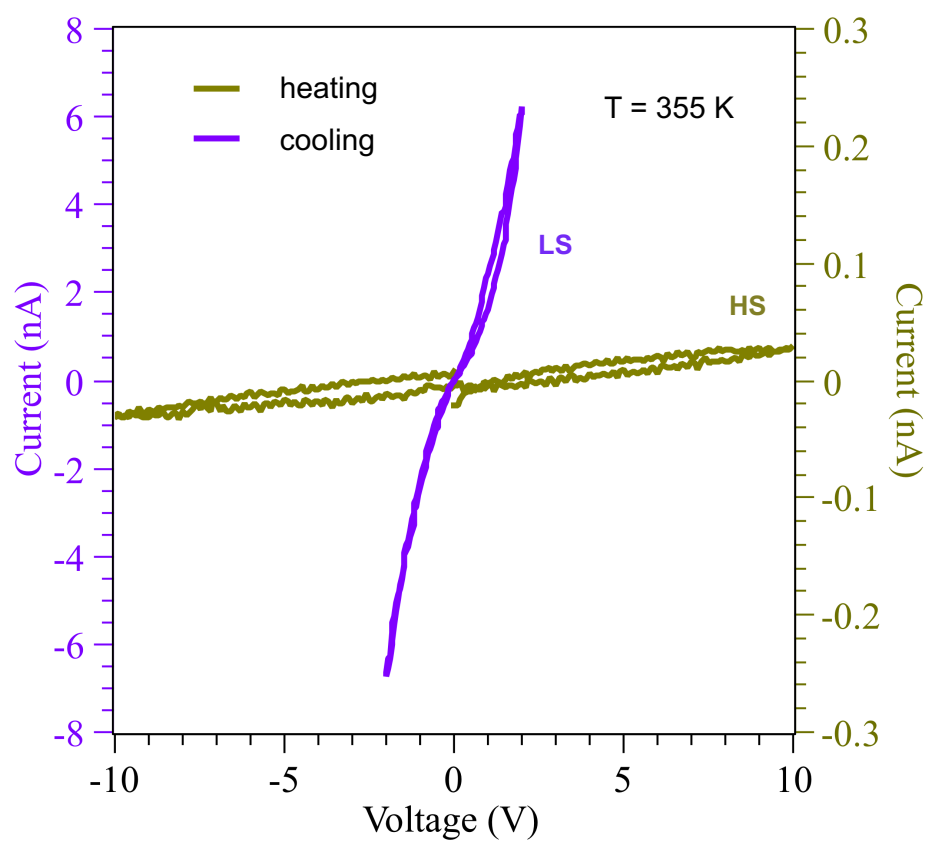

Figure S11. I-V characteristics of the device highlighted in the main text recorded within the thermal hysteresis region at $350 \mathrm{~K}$ in the heating (corresponding to the low-spin state) and cooling (high-spin state) mode. 
a)

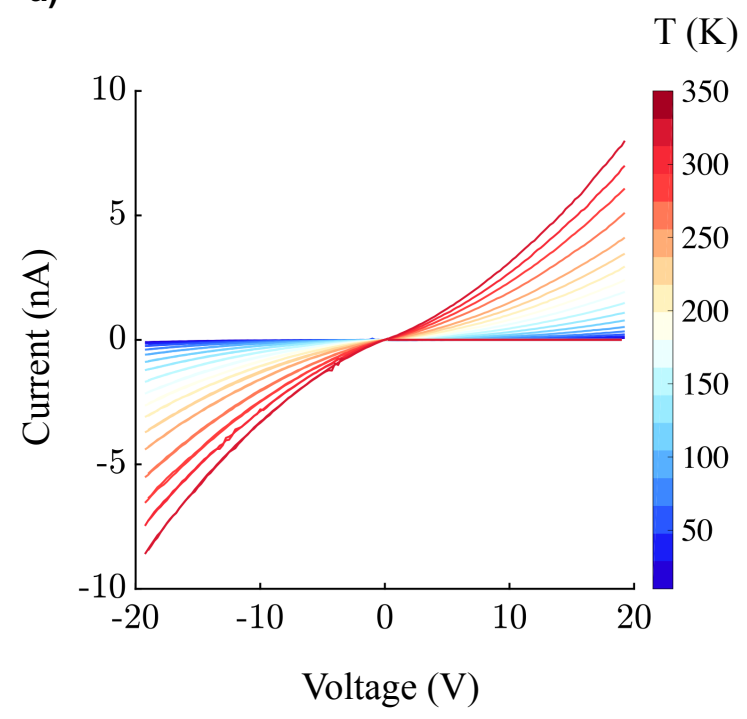

b)

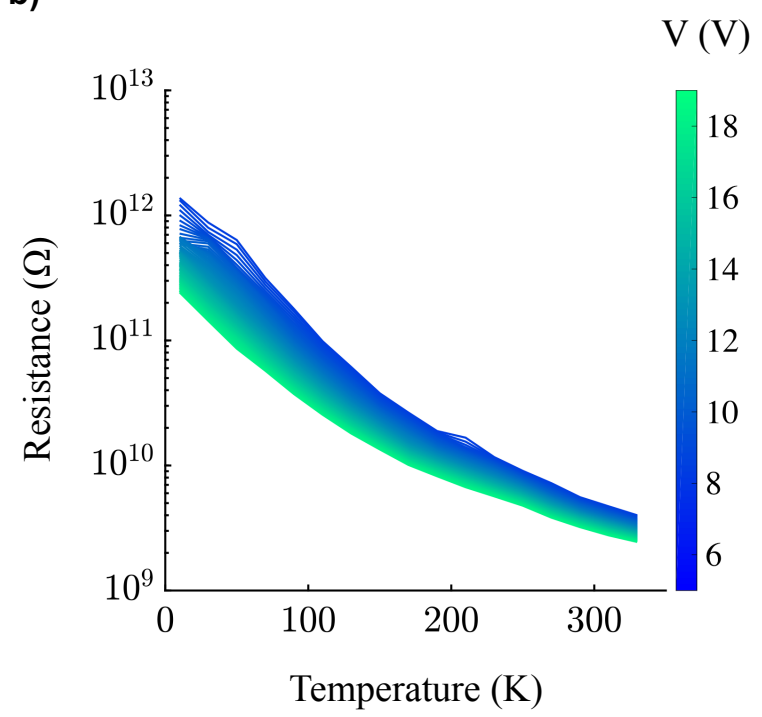

Figure S12. a) IV characteristics recorded on the fly while cooling down at $20 \mathrm{~K} \cdot \mathrm{min}^{-1}$ as a function of temperature. b) Corresponding resistance vs temperature characteristics at different bias voltages from 8 to $18 \mathrm{~V}$. The substantial increase in resistance upon decreasing temperature is typical of Coulomb blockade in core-shell NP arrays. 


\section{Numerical calculations}

To provide a better understanding of the charge transport mechanism, we modified the model from Devid et al. ${ }^{2}$ combining the Coulomb blockade regime and spin transitions through a simple macroscopic percolative network model. In brief, this model describes the influence of a spin transition on the device resistance $\mathrm{R}$ via a bond percolation model. We took into account the expected spin-state dependence and memory effect of the shell thickness and the relative dielectric permittivity (see Figure S12.b). This spin-state dependency will be considered in the calculation of the charging energy using $E_{\mathrm{C} \Sigma}=\mathrm{e}^{2} / 2 C_{\Sigma}$, where $C_{\Sigma}$ is the capacity of a NP surrounded by $N$ neighbors following ${ }^{3,4}$ :

$$
C_{\Sigma}=2 N \pi \varepsilon_{0} \varepsilon_{\mathrm{r}} r \ln (1+2 r / s)
$$

with $N=12$ (assuming a three-dimensional fcc packing), $s$ is the barrier length, and $r$ is the nanoparticle radius ( $r=10 \mathrm{~nm}$ and $s=4 \mathrm{~nm}$ gave qualitative agreement between experimental data and numerical results). The percolation model relates the device conductance G to the HS fraction amongst $\mathrm{Fe}^{\mathrm{II}}$ chains present at the shells in function of temperature $\chi_{H}^{\uparrow(\downarrow)}(T)$ (where $\uparrow$ $(\downarrow)$ means in the heating(cooling)modes, respectively) that is accompanied with a memory effect (see Figure S12.a). This can be achieved by leveraging the model developed by McLachlan, widely used in the literature when molecule-nanoparticle arrays are at play. ${ }^{5-7}$ Combining the percolation theory to the McLachlan's model allows to express the total conductance $G_{t o t}^{H S(L S)}$ of a highly disordered conductor network by relating it to the $\chi_{H}^{\uparrow(\downarrow)}(T)$ as such:

$$
\begin{gathered}
G_{t o t}^{H S(L S)}=G_{0}^{\uparrow(\downarrow)} \times G\left(\chi_{H}^{\uparrow(\downarrow)}(T)\right) \times e^{\left(-E_{c}^{\uparrow(\downarrow)} / T\right)} \\
G_{0}^{\uparrow(\downarrow)}=G_{0}^{L S}+\left(G_{0}^{H S}-G_{0}^{L S}\right) \times \chi_{H}^{\uparrow(\downarrow)}(T)
\end{gathered}
$$

Where $G_{0}^{L S}=1.25 \times 10^{-10} S$ and $G_{0}^{H S}=G_{0}^{L S} / 20$ are adjusted parameters to match experimental results.

$$
G\left(\chi_{H}^{\uparrow(\downarrow)}(T)\right)=\left\lfloor-b^{\uparrow(\downarrow)}-\sqrt{b^{\uparrow(\downarrow)^{2}}+4 \times \rho_{c} \times \gamma_{L S} \times \gamma_{H S} /-2 \rho_{c}}\right\rfloor^{4 / 3}
$$

Where $b^{\uparrow(\downarrow)}=\rho_{c} \times \gamma_{L S}-\gamma_{H S}+\chi_{H}^{\uparrow(\downarrow)}(T) \times\left(1+\rho_{c}\right) \times\left(\gamma_{L S}-\gamma_{H S}\right)$

For a packed and three-dimensional lattice of nanoparticles, $\rho_{c}=0.119$ is often used. ${ }^{8} \mathrm{We}$ adjusted the parameters $\gamma_{L S}$ and $\gamma_{H S}$ respectively to $20^{3 / 4}$ and 1 to match experimental curves. 
a)

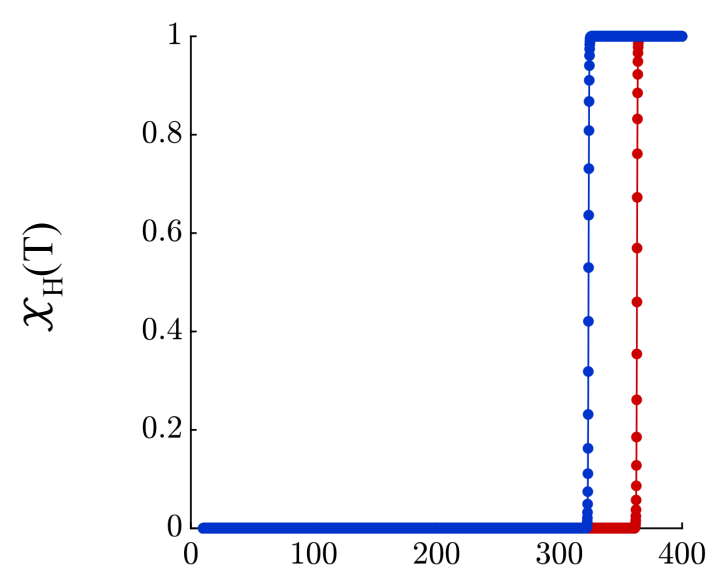

Temperature (K) b)

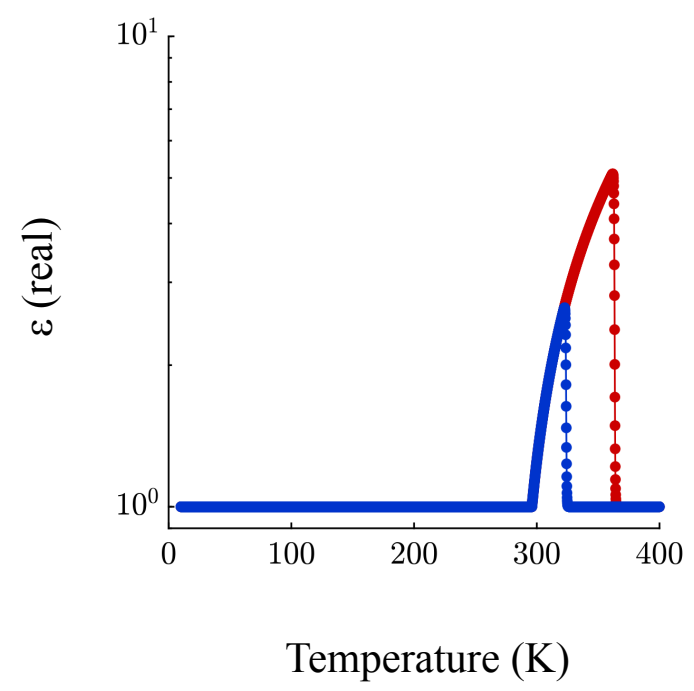

Figure S13. Simulated thermal hysteresis loop of the a) high spin fraction of $\mathrm{Fe}^{\mathrm{II}}$ chains composing the shells and $\mathbf{b}$ ) the real part recorded at $1 \mathrm{KHz}$ of the nanoparticle shell dielectric permittivity. ${ }^{9}$

Figure S13 presents a comparison of the experimental results and model calculations. The model captures the main observed experimental behaviour from low to high temperatures, including the clockwise direction of the hysteresis loops. This qualitative agreement between the present result and experiments lends credence to the other arguments pointing at transport through core-shell nanoparticle arrays in which thin insulating shells act as tunnel barriers.

The spin-state dependence of the dielectric permittivity $\varepsilon^{\uparrow(\downarrow)}$ as a function of the frequency of the electric field has been reported by Lefter et al. while investigating powder samples of the same compounds. ${ }^{10}$ They found that the SCO compound polarizability drops 5 times when switching from the LS to the HS state before reaching a value close to 1 . We also assumed a value of about 1 for lower temperatures below $300 \mathrm{~K}$ as to the best of our knowledge it had not been unveiled for this family of compounds.

$$
\varepsilon^{\uparrow(\downarrow)}=\varepsilon_{0}^{L S}+\left(\varepsilon_{0}^{H S}-\varepsilon_{0}^{L S}\right) \times \chi_{H}^{\uparrow(\downarrow)}(T)
$$

Where $\chi_{H}^{\uparrow, \downarrow}(T)=0.5+0.5 \times \tanh \left(\left(T-T^{\uparrow, \downarrow}\right) / w\right)$ with $\mathrm{w}$ equals to 0.5 , which acts on the steepness of the transition. The critical temperatures $T^{\uparrow, \downarrow}$ have been adjusted to match the best our experimental data. The numerical result of the high spin fraction in function of temperature employed in this model can be observed in Figure S12.a. From the temperature dependence of the high spin fraction $\chi_{H}^{\uparrow(\downarrow)}(T)$ we reproduced numerically the spin-state dependence of the 
dielectric permittivity of the 1D triazole-based SCO compound family employed in this work (see Figure S12.b). For this, we adjusted the following parameters:

$$
\begin{gathered}
\varepsilon_{0}^{L S}(T)=0.0625 \times T+(2.5-0.0625 * 320) \\
\varepsilon_{0}^{H S}=1
\end{gathered}
$$
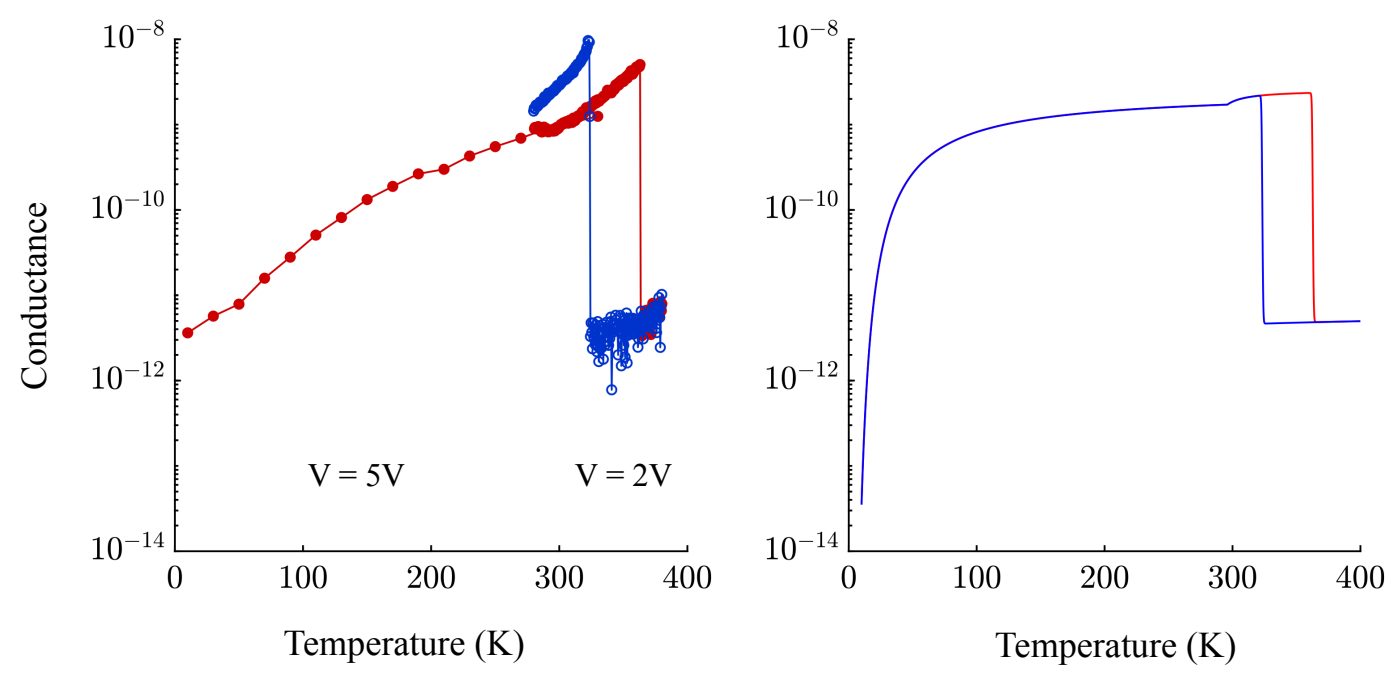

Figure S14. Comparison between model calculations and experimental results of the memory effect in the conductance versus temperature. (a) Data of conductance versus temperature selected from Fig. 4a for a voltage of 2 and $5 \mathrm{~V}$ for high and low temperatures respectively. (b) Calculated spin-state dependence of the conductance taking into account the dielectric properties of the spin-crossover nanoparticles and the respective volume change of the shells (estimated of about $6 \%$ greater in the HS state) ${ }^{10}$. The model captures the main features of the data, including the presence and the clockwise direction of the hysteresis loops. The red and blue lines and dots indicate heating and cooling mode, respectively.

\section{References:}

${ }^{1}$ J. Turkevich, Gold Bull. 1985, 18 (4), 125.

${ }^{2}$ E. J.Devid, P. N. Martinho, M. V. Kamalakar, I. Šalitroš, Ú. Prendergast, J. F. Dayen, V. Meded, T. Lemma, R. González-Prieto, F. Evers, T.E. Keyes, M. Ruben, B. Doudin, S. J. Van Der Molen, ACS Nano 2015, 9 (4), 4496.

${ }^{3}$ P. Beecher, A. J. Quinn, E. V. Shevchenko, H. Weller, G. Redmond, Nano Lett. 2004, 4 (7), 1289 .

${ }^{4}$ C. T. Black, C. B. Murray, R. L. Sandstrom, S. Sun, Science 2000, 290 (5494), 1131.

${ }^{5}$ J. Liao, S. Blok, S. J. Van Der Molen, S. Diefenbach, A. W. Holleitner, C. Schönenberger, A. 
Vladyka, M. Calame, Chem. Soc. Rev. 2015, 44 (4), 999.

${ }^{6}$ B. I. Shklovskii, A. L. Efros, Springer Verl. 1984.

${ }^{7}$ J. Wu, D. S. McLachlan, Phys. Rev. B 1997, 56 (3), 1236.

${ }^{8}$ K. H. Müller, J. Herrmann, B. Raguse, G. Baxter, T. Reda, Phys Rev B 2002, 66, 075417.

${ }^{9}$ C. Lefter, I. A. Gural'skiy, H. Peng, G. Molnár, L. Salmon, A. Rotaru, A. Bousseksou, P. Demont, Phys. Status Solidi Rapid Res. Lett. 2014, 8, 191.

${ }^{10}$ A. Grosjean, P. Négrier, P. Bordet, C. Etrillard, D. Mondieig, S. Pechev, E. Lebraud, J. F. Létard, P. Guionneau, Eur. J. Inorg. Chem. 2013, 796. 